\title{
Scalable Video Multicast over MANETs
}

\author{
Amir Asif, Uyen Trang Nguyen, and Guohua Xu \\ Department of Computer Science and Engineering \\ York University, Toronto, ON, Canada M3J 1P3 \\ Email: $\{$ asif, utn, gxu\}@cs.yorku.ca
}

\begin{abstract}
The paper presents a video multicasting architecture for mobile ad-hoc networks (MANETs). The proposed framework uses the scalable, noncausal predictive codec with vector quantization and conditional replenishment (SNP/VQR) for encoding video into multiple layers of data streams at bit rates between $10 \mathrm{~Kb}$ s to $500 \mathrm{~Kb}$ s. A rate adaptive multicast (RAM) routing algorithm is proposed, which dynamically adjusts the transmission rate based on the channel quality to obtain the optimum throughput. The scalability of SNP/VQR, coupled with RAM, enables strong resilience against bandwidth fluctuations as well as the capability to support a range of heterogeneous mobile receivers. Experimental results demonstrate that uninterrupted video of reasonable quality is multicasted with the proposed architecture even under heavy traffic conditions.
\end{abstract}

\section{INTRODUCTION}

Recent developments in the wireless technologies and portable computing coupled with demands for greater user mobility have led to the introduction of a new class of self deploying networking architecture referred to as mobile adhoc networks (MANETs). These networks are formed by a group of mobile nodes and require no fixed infrastructure, specialized routers, or centralized control. All mobile nodes function simultaneously as hosts and routers, and are capable of communicating with each other via packet radios. Although originally intended for military or disaster related operations, MANETs are becoming increasingly popular in short-term group communications such as symposiums and conferences for rapid sharing of multimedia information.

Due to their inherent broadcasting nature, MANETs are well suited for multicasting streaming video yet have not been fully utilized for such multimedia applications. Live video traffic has demands that are hard to be met by standard MANETs and improvements are required in the routing protocols, QoS provisioning, and error resilience tools of the video codecs. These issues arise primarily from low, widely fluctuating bandwidth capacity of the wireless channels and the limited battery power of the mobile nodes. The paper presents a framework for multicasting live video over MANETs. The bandwidth and power issues of MANETs are addressed as follows.

Limited Bandwidth: The video multicasting framework uses the scalable, noncausal predictive codec with vector quantization and conditional replenishment (SNP/VQR) [1], which offers superior video quality than the standard codecs including MPEG4 and H.264 at low bit rates. Use of SNP/VQR allows transmission at a lower rate than required by the standard

This work was supported in part by the Natural Science and Engineering Research Council (NSERC), Canada under Grant No. 228415. codecs with no perceivable difference in the video quality. To further overcome the bandwidth limitation, we employ the proposed rate-adaptive multicast (RAM) protocol to achieve higher packet delivery ratio and higher throughput.

Network/Receiver Heterogeneities: In order to accommodate a wide range of mobile devices, SNP/VQR offers scalable coding where the video is encoded into multiple layers of data streams. A subset of the compressed bit stream provides the base quality. Additional enhancement layers build on to the video quality in both the spatial and temporal domains. Receivers with powerful batteries and access to higher bandwidth channels can choose to receive all layers, therefore, acquiring video of the highest quality. To save energy and network bandwidth, other receivers may opt for a limited number of layers. The scalability of SNP/VQR also enables routers to prioritize transmissions by dynamically adjusting to the channel conditions and transmitting a reduced number of video layers at times of congestion.

Error Resilience: To conceal distortions introduced due to transmission errors, SNP/VQR uses spatial and temporal interpolation. A lost or damaged packet implies that certain blocks of the video can not be reconstructed accurately. SNP/VQR interpolates unavailable pixels from correctly received pixels in the neighboring blocks from the same and previous frames.

Concerning the organization of the paper, Sections II and III describe the main components of the proposed video multicasting architecture for MANETs. Performance evaluation of the system is included in Section IV, while concluding remarks are made in Section V.

\section{Proposed Video Multicasting Architecture}

Fig. 1 shows the main components of our multicasting scheme for real-time transmission of video in MANETs. A $\mathrm{SNP} / \mathrm{VQR}$ encoder [1] at the server compresses the video sequence frame by frame. A multicast sender, referred to as the video packetizer, transforms the compressed frame into an array of data packets, which are transmitted over the MANET. Because of the scalable nature of the codec, each subscriber has an option to select how many layers it wants to receive. After the selected layers are received, the SNP/VQR decoder reconstructs the compressied video frames. In case of transmission errors, SNP/VQR decoder applies error concealment techniques to the damaged pixels. A brief description of the main components of the proposed video multicasting scheme is included below. 


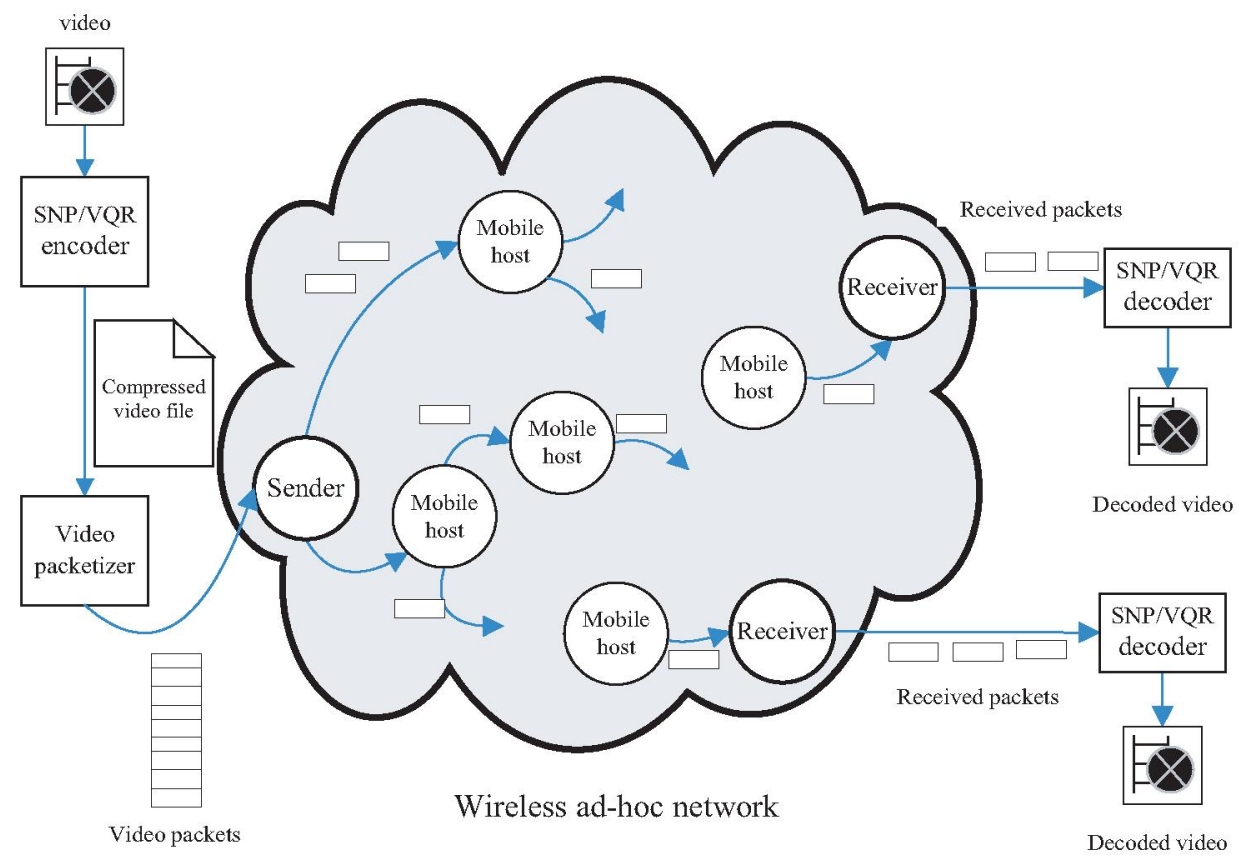

Fig. 1. Components of the real-time video transmission framework for MANETs

\section{A. SNP/VQR Codec}

As illustrated in Fig. 2, the compression procedure of $\mathrm{SNP} / \mathrm{VQR}$ has a predictive component followed by a quantization component. The predictive component, shown in part I of Fig. 2, has four stages as described below.

1) In the first stage, the vertical, horizontal, and temporal interactions $\left\{\beta_{v}, \beta_{h}, \beta_{t}\right\}$ are estimated from the input video $x(i, j, k)$ of dimensions $\left(N_{I} \times N_{J} \times N_{K}\right)$ by maximizing the likelihood function. The interactions define the state matrix $\mathcal{A}$ used to generate the error field as

$$
\begin{gathered}
\mathcal{A} \vec{X}=\vec{e} \\
\text { where } \mathcal{A}=I_{N_{K}} \otimes A_{1}+H_{N_{K}}^{1} \otimes A_{2}, \\
A_{1}=I_{N_{I}} \otimes B+H_{N_{I}}^{1} \otimes C, \text { and } A_{2}=I_{N_{I}} \otimes D .
\end{gathered}
$$

Vectors $\vec{X}$ and $\vec{e}$ represent, respectively, the row-ordered pixels of the input video $x(i, j, k)$ and the 3D whitened error field $e(i, j, k)$ for the entire sequence. In Eqs. (2)(3), the symbols $I_{N_{K}}$ and $I_{N_{I}}$ are identity matrices, while $H_{N_{K}}^{I}$ and $H_{N_{I}}^{I}$ are Toeplitz matrices that have zeros everywhere except for the first upper and lower diagonals, which are composed of all ones. The subscript denotes the order of the matrix. The operator $\otimes$ represent the Kronecker product and the constituent blocks are

$B=-\beta_{h} H_{N_{J}}^{1}+I_{N_{J}}, C=-\beta_{v} I_{N_{J}}$ and $D=-\beta_{t} I_{N_{J}}$.

The interaction parameters are also required at the decoder to reconstruct the video and constitute overhead information transmitted to the receiver.

2) In the second stage, the 3-D noncausal model, Eq. (1), is transformed to an equivalent unilateral representation that uses block triangular matrices $\{L, F\}$ for prediction. The recursive form is obtained by lower Cholesky decomposition $\mathcal{A}=\mathcal{L}^{T} \mathcal{L}$, where $\mathcal{L}$ is given by

$$
\mathcal{L}=\left[\begin{array}{ccccc}
L^{(1)} & \underline{0} & . & . & \underline{0} \\
F^{(2)} & L^{(2)} & \underline{0} & . & \underline{0} \\
. & \ddots & \ddots & . & . \\
\underline{0} & \cdot & F^{\left(N_{K}-1\right)} & L^{\left(N_{K}-1\right)} & \underline{0} \\
\underline{0} & \cdot & \underline{0} & F^{\left(N_{K}\right)} & L^{\left(N_{K}\right)}
\end{array}\right]
$$

The forward Cholesky blocks $L^{(k)}$ 's in $\mathcal{L}$ are lower triangular and $F^{(k)}$ 's are upper triangular.

3) Stages three and four generate the uncorrelated error field by subtracting the values of the pixels in the predicted frame from the original pixel values. In SNP/VQR, we combine stages 3 and 4 such that the whitened error field $\vec{v}=\mathcal{L}^{-T} \vec{e}$ is obtained separately for each frame $k$ from the input video $x(i, j, k)$ by transforming Eq. (1) into the following unilateral model

$$
\begin{aligned}
L^{(1)} X^{(1)} & =v^{(1)} \\
F^{(k)} X^{(k-1)}+L^{(k)} X^{(k)} & =v^{(k)},\left(2 \leq k \leq N_{K}\right),
\end{aligned}
$$

where $X^{(k)}$ and $v^{(k)}$ represent the row-ordered pixels of the original video $x(i, j, k)$ and the whitened error field $v(i, j, k)$ in frame $k$. Since the noncausal prediction at the receiver is based on the reconstructed frames, the encoder also uses the reconstructed frames for prediction.

To achieve high compression ratios, the whitened error video $v^{(k)}$ is vector quantized using an $N$-stage cascaded vector quantization (VQ). The VQ step is shown in part II of Fig. 2, where we also apply conditional replenishment at each stage 


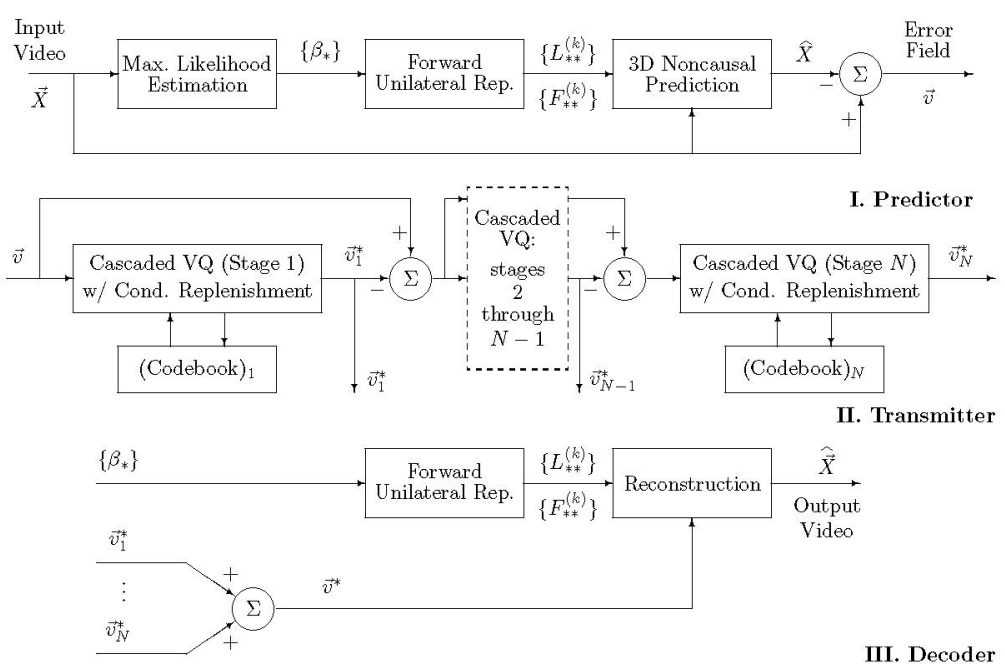

Fig. 2. Block Diagram Representation of the SNP/VQR video codec.

of cascaded VQ by encoding and transmitting the vector quantized block only if its index is different from the corresponding index at the same location in the previous frame. Conditional replenishment leads to considerable reduction in the number of code vectors transmitted. The indices of the vector quantized blocks $\left\{\vec{v}_{1}^{*}, \ldots, \vec{v}_{N}^{*}\right\}$ constitute the output of the VQ step and are transmitted over the channel. The vector quantized blocks $\vec{v}_{1}^{*}$ obtained from stage 1 of the cascaded VQ form the base layer of the streaming video, while the blocks $\left\{\vec{v}_{2}^{*}, \ldots, \vec{v}_{N}^{*}\right\}$ form $(N-1)$ additional enhancement layers that add to the video quality obtained from the base layer. Scalability makes SNP/VQR suitable for multicasting live video over MANETs.

Part III of Fig. 2 represents the SNP/VQR decoder, which reconstructs the video from the VQ blocks $\left\{\vec{v}_{1}^{*}, \ldots, \vec{v}_{N}^{*}\right\}$ by inverting the steps of the encoder (part I of Fig. 2) in the reverse order. Since the VQ step introduces controlled distortion, the reconstructed video is not a perfect match of the input video $\vec{X}$. As in the standard codecs, SNP/VQR uses lossy compression to achieve low bit per pixel representations but the subjective quality of the reconstructed video is much superior to these standards. At bit rates below $150 \mathrm{Kbps}$, MPEG4 and H.263 exhibits several visual degradations such as blocking. SNP/VQR exhibits better visual quality with no blocking and more details are retained in the compressed video. In terms of peak signal to noise ratio (PSNR), SNP/VQR outperforms MPEG4 by $1 \mathrm{~dB}$ and $\mathrm{H} .263$ by about $1.5 \mathrm{~dB}$ at transmission rates around $50 \mathrm{Kbps}$. See [1] for more details.

\section{B. Bandwidth Adaptation / Layer Coding}

SNP/VQR achieves scalability in both the spatial and temporal domains. The spatial scalability is a direct consequence of cascaded VQ used to compress the prediction error. In the experimental setup, we use 3-stage, 6-bit VQ with a 321-bit distribution between the three stages. For best spatial quality, the outputs of all three stages are transmitted to the receiver. The intermediate quality uses the outputs from stages 1 and 2 of $\mathrm{VQ}$, while the lowest quality uses the output from stage 1 .

The frame rate scalability is achieved by representing video $X^{(k)},\left(1 \leq k \leq N_{K}\right)$, in three layers. The base layer consists of every fifth frame, $X^{(4 k-3)}$, and is transmitted to all receivers. The first enhancement layer encodes intermediate frames, $X^{(4 k-1)}$, while the second enhancement layer encodes the remaining frames, $X^{(2 k)}$. To fully exploit the temporal redundancy, prediction in the first enhancement layer uses frames reconstructed from the base layer as well as original frames $X^{(4 k-1)}$. Similarly, prediction in the second enhancement layer uses frames reconstructed from the base and first enhancement layers in addition to original frames $X^{(2 k)}$.

By combining the spatial and frame rate scalabilities, SNP/VQR offers three qualities of services: Gold, Silver, and Bronze, at bit rates between $10 \mathrm{Kbps}$ to $500 \mathrm{Kbps}$. The bronze service uses the base layer of the temporal feed compressed with the first stage of the cascaded VQ. The silver service couples the base and first enhancement layers compressed using the first two stages of VQ, while the gold service uses all three temporal layers compressed with the 3-stages of VQ.

Using the above scalability feature, SNP/VQR allows decoding at multiple rates from the same bit stream. Bandwidth adaptation can be performed either by the sender or by the intermediate routers. A sender may use different stages of the cascaded VQ and select to transmit some or all of the enhancement layers depending on the network conditions. Conversely, a router may choose to drop packets of the least important layer(s) on detecting the onset of congestion.

\section{Error Concealment}

To conceal distortion introduced due to transmission errors, SNP/VQR uses spatial and temporal interpolation. A lost or damaged packet in SNP/VQR implies that certain blocks of the error image $\vec{v}$ are not available for the reconstruction of the video. Unavailable error pixels in these damaged blocks are interpolated from the correctly received pixels in adjacent 
blocks from the same and/or previous frames of the error image $\vec{v}$. We consider the following error concealment methods:

- Median concealment: This spatial concealment method replaces the error pixels $v(i, j, k)$ corresponding to a lost VQ block with the median value of the error pixels in its four adjacent neighboring blocks (above, below, left and right) if at least one of them is received correctly. Else, the unavailable error pixels are filled with zeros.

- Mean concealment: Same as median concealment, except that the mean value is used instead of the median.

- Temporal concealment: The error pixels $v(i, j, k)$ within a lost VQ block is filled up with the error pixel values at the corresponding location in the previous frame.

Our experimental results show that the concealment methods enhance the video quality. The temporal concealment performs slightly better than the other two approaches due to high similarity between successive video frames. We use temporal concealment in our multicasting framework with the following modification. To facilitate interpolation, SNP/VQR interleaves the VQ indices during packetization so that loss of one packet does not lead to loss of contiguous blocks.

\section{NeTworking PRoToCols}

In this section, we discuss the networking protocols used in our video multicasting framework.

\section{A. Layered Multicast}

Common approaches for multicasting scalable video include priority packet dropping (PPD) [2] and receiver-driven layered multicast (RLM) [3]. PPD prioritizes the transmission of the packets based on the originating video layer. Packets derived from the base layer have the highest priority for transmission, where as the upper enhancement layers' packets are given progressively lower priorities. PPD requires routers to implement a priority-based packet scheduling policy where the lower-priorities packets are dropped at times of congestion. In comparison, RLM transmits packets from different video layers over separate multicast groups. A receiver probes for the available bandwidth by periodically joining a higher layer group and estimates the packet loss rate. If the packet loss exceeds a predefined threshold, the receiver leaves the latest experimented group. Otherwise, it stays at the new subscription level. RLM is unsuitable for layered multicast in MANETs due to the following reasons. First, the joining experiments add adversely to the network traffic, especially when the size of the multicast group is large. Second, estimating the available bandwidth in a bursty network is difficult. Finally, PPD is simpler to implement compared to RLM. Therefore, we chose PPD in our video multicast framework.

\section{B. Multicast Routing}

We implement the on-demand multicast routing protocol (ODMRP) in our framework since it provides higher packet delivery ratio and throughput than most other multicast routing protocols [4]. ODMRP sources periodically update routing tables and membership information by flooding the network with route refresh packets called JOIN_QUERY. When a node receives a non-duplicate JOIN_QUERY, it stores the upstream node ID (i.e., backward learning) and rebroadcasts the packet. When the JOIN_QUERY packet reaches a multicast receiver, the receiver creates a JOIN_REPLY packet and broadcasts it to its neighbors. When a node receives a JOIN_REPLY, it checks if the next node ID of one of the entries matches its own ID. If it does, the node realizes that it is on the path to the source and thus is part of the forwarding group. It then broadcasts its own JOIN_REPLY built upon the matched entries. The JOIN_REPLY packet is thus propagated by each forwarding group member until it reaches the multicast source via the shortest path. This process constructs (or updates) the routes from sources to receivers and builds a mesh of nodes, referred to as the forwarding group. The periodic route refreshment mechanism used by ODMRP also allows us to implement the rate adaptive multicast algorithm, which is discussed next.

\section{Rate Adaptive Multicast}

A current trend in wireless communications is to enable wireless hosts to transmit at different rates. For example, IEEE 802.11 b specifies transmission rates of $1 \mathrm{Mbps}, 2 \mathrm{Mbps}$, $5.5 \mathrm{Mbps}$ and $11 \mathrm{Mbps}$. Rate adaption is the process of dynamically switching between the transmission rates based on channel conditions to obtain optimum throughput. Existing rate-adaptation protocols are proposed for unicast communications [5], [6] and cannot be applied directly to multicast. We propose a rate-adaptive multicast (RAM) routing algorithm that is based on ODMRP. Following is an overview of RAM, which uses a parameter referred to as the weight of the sourceto-destination path. Given the optimal transmission rate $R$ of a link (as determined by the signal strength of a received packet), the weight of the link is defined as $1 / R$. The weight of a path is the sum of the weights of all links on that path.

In the RAM protocol, we use JOIN_QUERY packets of ODMRP to estimate the channel conditions and to record the weights of the paths traversed by these packets. The weights are recorded in a field referred to as pathWeight. After receiving a JOIN_QUERY, every node on a path between the sender and receiver, measures the signal strength and suggests a rate, SuggestedRate, derived from the signal strength using an algorithm similar to RBAR [5]. The SuggestedRate is converted to the link weight, which is then added to the pathWeight recorded in the JOIN_QUERY.

A node participating in the route discovery/refresh process may receive multiple JOIN_QUERY packets from the same sender that have arrived from different paths. The node considers all of these JOIN_QUERY packets and selects the path with the minimum pathWeight value. If the node is a receiver, the selected path is the source-to-destination path with the lowest total transmission time among those considered. The receiver then creates a JOIN_REPLY that records the routing information and sends the JOIN_REPLY to the source, as in the ODMRP algorithm. To prevent the implosion of the JOIN_REPLY packets, every node on paths from the receivers to the sender consolidates information from several 
TABLE I

DISTRIBUTION OF VIDEO PACKETS LOST IN THE SIMULATED MANET

\begin{tabular}{|l|c|c|c|c|c|}
\hline \multirow{2}{*}{ Layer } & \multirow{2}{*}{ Packets } & \multicolumn{2}{|c|}{$\begin{array}{c}\text { Packets Lost in } \\
\text { Light Traffic }\end{array}$} & \multicolumn{2}{c|}{$\begin{array}{c}\text { Packets Lost in } \\
\text { Heavy Traffic }\end{array}$} \\
\cline { 3 - 6 } & Number & Rate & Number & Rate \\
\hline \hline Bronze & 4,880 & 70 & $1.43 \%$ & 196 & $4.02 \%$ \\
\hline Silver & 4,880 & 99 & $2.03 \%$ & 320 & $6.56 \%$ \\
\hline Gold & 4,800 & 175 & $3.65 \%$ & 3,276 & $68.25 \%$ \\
\hline Total & 14,400 & 344 & $2.70 \%$ & 3,795 & $26.28 \%$ \\
\hline
\end{tabular}

JOIN_REPLY packets it receives in the downstream direction (i.e., from a sender towards a receiver) into one JOIN REPLY, as in ODMRP. If a node is on the selected path, it uses the SuggestedRate recorded earlier for transmitting data packets at the physical layer. Although the rate information is updated periodically, the recorded rates may become inaccurate before the next route refresh round due to the mobility of the nodes. Still, these rate values offer better performance than single-rate multicast as is shown experimentally in [7].

\section{EXPERIMENTAL RESULTS AND DISCUSSION}

The performance of our video multicast framework was tested for several video sequences under different networking conditions. Here, we present results from one set of experiments, which transmit the carphone sequence over a 50-node MANET, simulated using the QualNet software [8]. The nodes move randomly at a speed of $1 \mathrm{~m} / \mathrm{s}$ in an area confined to $(1000 \times 1000)$ square meters. Each node has a maximum queue size of 50 Kbytes. An arbitrary node, selected as the server, initiates the video session that can serve up to a maximum of 20 additional receivers. A random number generator between 1 and 50 is used to select the twenty receiving nodes. The receivers join the multicast group at the beginning of the video session and stay on till the end of transmission. Once the multicast group is formed, the server compresses the video in real time and classifies the resulting packets in three categories: gold, silver and bronze depending on the video layer from which the packet has originated. Packets from the three categories are transmitted over three different multicast groups. The transmitting rate for each group is 60 packets/sec, which equals the output rate of the SNP/VQR encoder. Prioritized transmission is implemented at every mobile host using several queues with different priorities. At times of congestion, packets are discarded using the PPD algorithm with first-infirst-out scheduling. The multicast routes are refreshed using the ODMRP algorithm with the refresh interval set to $20 \mathrm{~s}$.

To simulate the wireless channels, a two-ray propagation model is used with the free space path loss set to $(2.0,0.0)$ for near-sight and the plane earth path loss of $(4.0,0.0)$ for farsight. The transmission power for each node is set at $15 \mathrm{~dB}$. The propagation range for the radio signal originating from a node is $250 \mathrm{~m}$. We implemented PHY $802.11 \mathrm{~b}$ at the physical layer, which uses a preconfigured bit error rate based packet reception model. The MAC802.11 with Distributed Coordination Function (DCF) is chosen as the medium access control protocol. Multicast and broadcast transmissions use carrier sensitive
TABLE II

AVERAGE PSNR'S FOR BRONZE, SILVER, AND GOLD SERVICES.

\begin{tabular}{|l|c|c|c|c|}
\hline Network & Packets & \multicolumn{3}{|c|}{ Quality of Service } \\
\cline { 3 - 5 } Conditions & Lost & Bronze & Silver & Gold \\
\hline \hline Ideal (no loss) & $0 \%$ & $28.75 \mathrm{~dB}$ & $29.58 \mathrm{~dB}$ & $29.99 \mathrm{~dB}$ \\
\hline Light traffic & $2.70 \%$ & $28.59 \mathrm{~dB}$ & $29.32 \mathrm{~dB}$ & $29.69 \mathrm{~dB}$ \\
\hline Heavy traffic & $26.28 \%$ & $28.36 \mathrm{~dB}$ & $28.88 \mathrm{~dB}$ & $29.00 \mathrm{~dB}$ \\
\hline
\end{tabular}

multiple access with collision avoidance (CSMA/CA). In addition to CSMA/CA, unicast transmissions also use the request to send/clear to send (RTS/CTS) algorithm.

To evaluate the effectiveness of the priority packet dropping scheme, we measure the rate of packet losses for the three video layers under both light and heavy traffic conditions. The results are shown in Table I, where the number of lost packets averaged over the twenty receivers is included. As expected, the packets loss rates under light traffic are low, while the packet loss rates under heavy traffic are high. Under heavy traffic conditions, the majority of the lost packets are from the gold category. This shows that priority dropping of packets works effectively, since the gold category includes video data from the highest enhancement video layer

The quality of reconstructed video frames is assessed by computing the average peak SNR (PSNR), defined as

$$
\text { Average PSNR }=\frac{1}{R \times N_{K}} \sum_{r=1}^{R} \sum_{k=1}^{N_{K}} \operatorname{PSNR}(k, r),
$$

where $\operatorname{PSNR}(k, r)$ is defined as the PSNR of frame $k$ reconstructed by receiver $r, N_{K}$ is the total number of frames in the video sequence, and $R$ is the number of receivers included in the multicast session. Table II lists the average PSNR's under three different network conditions: no packet loss, minimal packet loss under light traffic, and moderate packet loss under heavy traffic. We observe that the PSNR's of the reconstructed videos for the bronze service is similar across the three traffic conditions. Since PPD seeks to retain most of the packets from the base layer, heavy traffic conditions do not adversely affect the quality of the video at the bronze level. Compared to the ideal setting with no packet loss, the PSNR of the gold service drops by $0.3 \mathrm{~dB}$ under light traffic and by about $1 \mathrm{~dB}$ under heavy traffic. Conceivably, this significant decrease in the PSNR's is due to the large number of gold packets being dropped under heavy traffic conditions. Finally, we observe that the gold service provides a higher PSNR than the bronze service even under heavy traffic conditions.

To provide subjective evaluation, Fig. 3 includes several frames reconstructed by the SNP/VQR decoder at different receivers. Since the quality of the reconstructed video varies from one receiver to another, we chose frames from video sequences whose average PSNR is closest to the PSNR values provided in Table II. The sample frames demonstrate that SNP/VQR, combined with the proposed networking protocols, offers good perceptual visual quality even under congested conditions. Future work will focus on implementing the proposed multicasting architecture in a real wireless environment. 


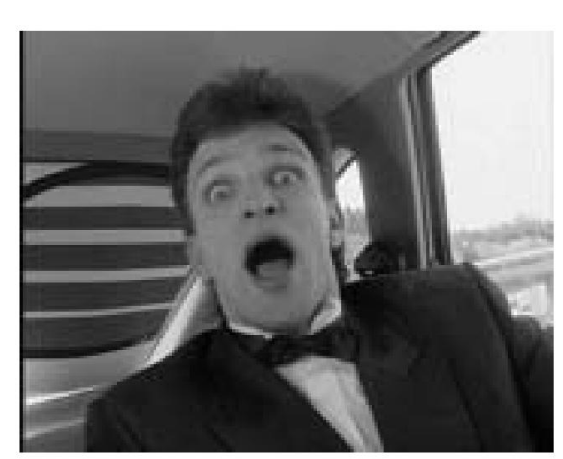

(a) Original frame

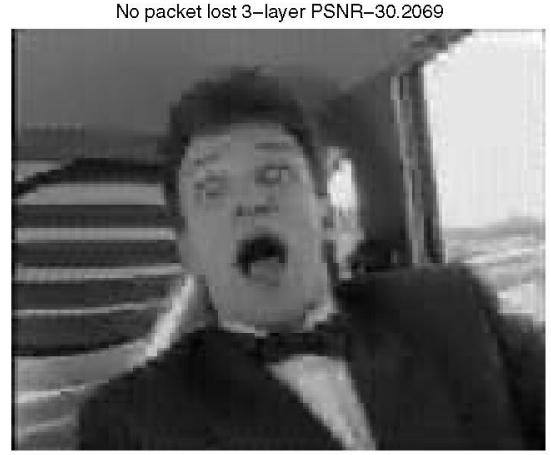

(b) No packet loss - Gold service

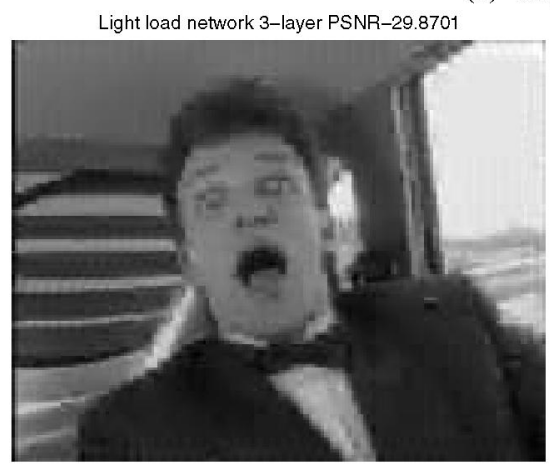

(c) Gold service

Heavy load network 3-layer PSNR-29.4976

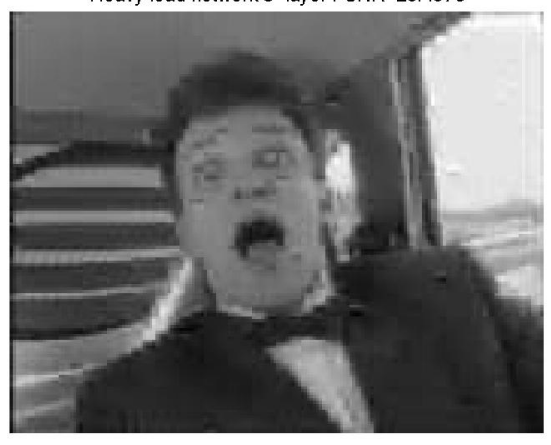

(f) Gold service

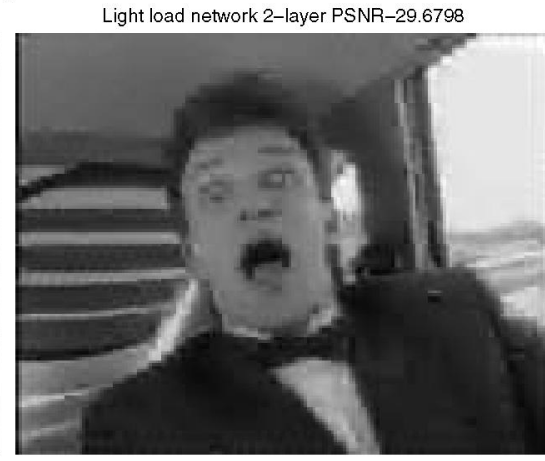

(c)-(e): Light traffic conditions

(d) Silver service

Heavy load network 2-layer PSNR-29.3802

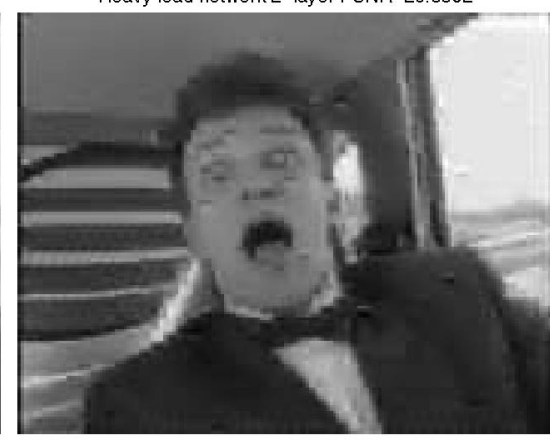

(f)-(h): Heavy traffic conditions

(g) Silver service

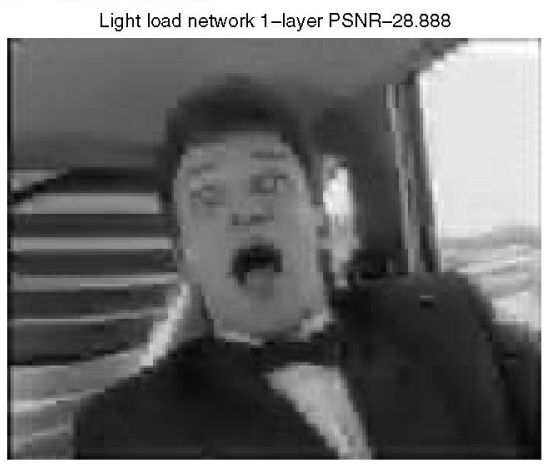

(e) Bronze service Heavy load network 1-layer PSNR-28.686

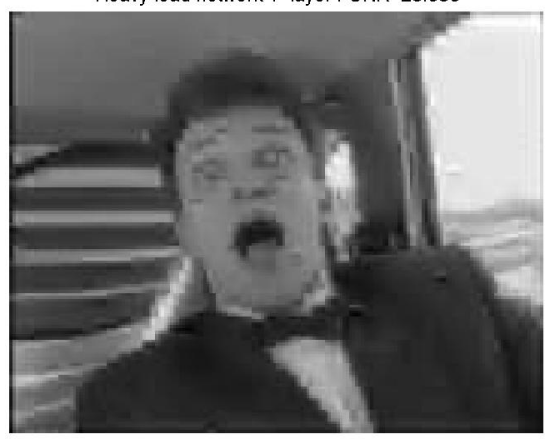

(h) Bronze service

Fig. 3. Reconstructed frames obtained using bronze, silver and gold services under light and heavy traffic conditions.

\section{SUMMARY}

A scalable video codec, SNP/VQR, and the supporting networking protocols for multicasting real time, streaming video over MANETs are presented. To support heterogeneous receivers, $\mathrm{SNP} / \mathrm{VQR}$ encodes video into multiple layers of data streams. The scalability feature of SNP/VQR, coupled with our rate adaptive multicast algorithm, enables routers to dynamically adjust the transmission rates based on the channel conditions. In our experiments, the proposed architecture offers reasonable quality of uninterrupted video under heavy traffic.

\section{REFERENCES}

[1] A. Asif and M. G. Kouras, "Scalable Video Codec by Noncausal Prediction, Cascaded Vector Quantization, and Conditional Replenishment,"
IEEE Trans. on Multimedia, Feb. 2006, vol. 8, no. 1, pp. 19-31.

[2] B. J. Vickers, C. Albuquerque, and T. Suda, "Source-Adaptive Multilayered Multicast Algorithms for Real-Time Video Distribution," IEEE/ ACM Trans. on Networking, Sep. 2000, vol. 8, no. 6, pp. 720-33.

[3] S. McCanne, V. Jacobson, and M. Vetterli, "Receiver-driven Layered Multicast," ACM SIGCOMM, Aug. 96, vol. 26(4), pp. 117-30.

[4] S. J. Lee, W. Su, J. Hsu, M. Gerla, and R. Bagrodia, "A Performance Comparison Study of Ad Hoc Wireless Multicast Protocols," Proceedings of IEEE INFOCOM, Tel Aviv, Israel, 2000, vol. 2, pp. 565-74.

[5] G. Holland, N. H. Vaidya and P. Bahl, "A Rate-Adaptive MAC Protocol for Multi-Hop Wireless Networks," Proc. of ACM MOBICOM, 2001.

[6] J. Pavon and S. Choi, "Link Adapt. Strategy for IEEE 802.11 WLAN via Received Signal Strength Measurement," Proc. of IEEE ICC, 2003.

[7] U. T. Nguyen and X. Xiong,"Rate-adaptive Multicast in Mobile Adhoc Networks," IEEE Int. Conf. on Wireless and Mobile Computing, Networking and Communications 2005 (WiMob 2005), Aug. 2005.

[8] QualNet Network Simulator, http://www.scalable-networks.com/. 